prescriber PGT perceptions and current parameters and barriers for use. Follow-up surveys were administered 3 months post-implementation. Project processes were measured by assessing the rate of medication failure template usage, as well as thePGT EHR upload rate.

RESULTS: A comparison of baseline and follow-up surveys indicated there was little change in prescriber view of test utility, receptiveness, and likelihood of use. This may be attributed to previous experience with testing and to PGT manufacturer education. View of parameters and barriers for use did change. Key parameter for use changes included patient experience of adverse reaction (increase) and only 2 medication failures from the same class (decrease). Key barrier to use changes included time to results (decrease). 3 PGT were completed during the project. All patients met the protocol criteria for testing. None of these patients had medication failures documented using theEHR template; all of the patients did have documentation using each prescriber's preferred method. 2 of the 3 tests were uploaded to the EHR. The first test completed was not integrated, likely due to support staff becoming accustomed to the new workflow. 117 historical PGT were also integrated into the EHR.

CONCLUSIONS: While 16 to $20 \%$ of the population meets the criteria for MDD, available treatments achieve symptom remission only $40 \%$ of the time (Singh, 2014). Patients who do not achieve remission experience relapse more quickly and are more likely to develop chronic non-remitting MDD (Gaynes, 2016). While the PGT evidence base is still evolving, its use in clinical practice has the potential to improve depression treatment outcomes. This study highlighted continued barriers to PGT use in a practice setting, while implementing key interventions, including PGT use guidelines and EHR integration, to improve its systematic and appropriate use.

FUNDING ACKNOWLEDGEMENTS: No funding.

152

\section{Pimavanserin for the Treatment of Parkinson's Disease Psychosis: Number Needed to Treat, Number Needed to Harm, and Likelihood to Be Helped or Harmed}

Leslie Citrome, MD, MPH'; James Norton, $P h D^{2}$; Kathy Chi-Burris, $\mathrm{MPH}^{3}$; and George Demos, $\mathrm{MD}^{4}$

${ }^{1}$ Clinical Professor or Psychiatry and Behavioral Sciences, Department of Psychiatry \& Behavioral Sciences, New York Medical College, Valhalla, NY
${ }^{2}$ Sr. Director Medical Affairs, ACADIA

Pharmaceuticals, Inc. San Diego, CA

${ }^{3}$ Sr. Director Biostatistics, ACADIA Pharmaceuticals, Inc. San Diego, CA

${ }^{4}$ Executive Director Drug Safety and Pharmacovigilance, ACADIA Pharmaceuticals, Inc. San Diego, CA

ABSTRACT: Study Objective: Psychosis is common in Parkinson's disease (PD) and increases in both frequency and severity with disease duration. It is associated with increased morbidity/mortality, complicates management of motor symptoms and often leads to long-term care placement. Pimavanserin (PIM) is a highly selective serotonin 5-HT2A receptor antagonist/inverse agonist indicated for the treatment of hallucinations and delusions associated with PD psychosis (PDP). The study aim is to review theevidence-base for PIM for the treatment of PDP using the metrics of evidence-based medicine, namely number needed to treat (NNT), number needed to harm $(\mathrm{NNH})$, andlikelihood to be helped or harmed $(\mathrm{LHH})$, in order to better place this intervention into clinical perspective.

METHODS: NNT and NNH are measures of effect size and indicate how many patients would need to be treated with one agent instead of the comparator in order to encounter one additional outcome of interest. A useful medication is one with a low NNT and a high NNH when comparing it with another intervention; a low NNT and a high NNH would mean one is more likely to encounter a benefit than a harm. Categorical efficacy and tolerability data was extracted from the clinical trial databases of the double-blind placebo-controlled studies of PIM in persons with PDP. The studies were 6 weeks in duration and fixed dose with the exception of study ACP-103-006 which was 4-weeks in duration. NNT and NNH values were calculated with their respective $95 \%$ confidence intervals. Efficacy endpoints were defined based on 2 definitions: a) Scale for the Assessment ofPositive Symptoms in Parkinson's Disease (SAPS-PD) total score decrease $\geq 3$ points from baseline and b) Clinical Global Impressions-Improvement scale (CGI-I) score of 1 (very much improved) or 2 (much improved). Tolerability outcomes of clinical interest, occurring at any time in available studies were assessed, including discontinuation due toan adverse event (AE). Likelihood to be helped or harmed (LHH) was then calculated contrasting therapeutic response vs. discontinuation because of an $\mathrm{AE}$.

RESULTS: NNT values for PIM $34 \mathrm{mg} / \mathrm{d}$ vs. placebo for several definitions of clinical response are $<10$, and as robust as 4 , denoting that PIM is a potentially efficacious intervention. $\mathrm{NNH}$ values for tolerability outcomes for PIM $34 \mathrm{mg} / \mathrm{d}$ (as well as for doses that range from $8.5 \mathrm{mg} / \mathrm{d}$ to $51 \mathrm{mg} / \mathrm{d}$ ) are $>10$, and/or 
are not statistically significant, and/or show an advantage for PIM over placebo (such as for postural hypotension), denoting that PIM is a potentially tolerable intervention. In terms of LHH, PIM $34 \mathrm{mg} / \mathrm{d}$ is about 5 times more likely to result in clinical response (as measured by $\geq 3$ point decrease from baseline on the SAPS-PD) vs. discontinuation due to an adverse event.

CONCLUSIONS: Using the metrics of NNT, NNH, and LHH, PIM $34 \mathrm{mg} / \mathrm{d}$ for the treatment of PDP appears to have a compelling benefit-risk profile.

FUNDING ACKNOWLEDGEMENTS: Clinical study was funded by ACADIA Pharmaceuticals Inc.

\section{5}

\section{Improvement of VIIIth Cranial Nerve Function With Cariprazine}

\author{
Maximous K. Philobos, MS4 ${ }^{1}$; and Alan R. Hirsch, $M D^{2}$ \\ ${ }^{1}$ Avalon University School of Medicine, Willemstad, \\ Curacao \\ ${ }^{2}$ Smell and Taste Treatment and Research Foundation, \\ Chicago, IL
}

ABSTRACT: Introduction: Cariprazine an atypical antipsychotic which acts as a dopamine D3-prefering partial agonist at dopamine D2/D3 receptors, as an antagonist at over stimulated dopamine receptors, as a partial agonist at serotonin 5-HT1A receptors, and as an antagonist of 5-HT2A receptors (Citrome, 2016; Kiss, 2010). While indicated for the treatment of schizophrenia and bipolar disorder, it has never been described to improve disorders of cranial nerve VIII. A patient with hearing loss associated with tinnitus, responsive to cariprazine, is reported.

METHODS: Case Study: A 34 year old right handed married male 5 years prior to presentation developed bilateral auditory hallucinations of whispers, and one male disparaging voice. Approximately 6 months later it began belittling him, whereupon he was diagnosed with schizoaffective disorder, attention deficit hyperactivity disorder, and obsessive-compulsive disorder.

Three months prior to presentation he developed sepsis and became comatose. Upon awakening he experienced constant tinnitus, AS more than $\mathrm{AD}$ high pitched, without diurnal variation, which has been unrelenting. Coincident with the tinnitus was decreased hearing AS more than $\mathrm{AD}$. Within a few days of treatment with cariprazine at $1.5 \mathrm{mg}$ a day, the tinnitus transiently resolved and after raising the cariprazine to $3 \mathrm{mg}$ per day the tinnitus abruptly stopped and his hearing returned to normal after 2 months. One and a half days after discontinuing the cariprazine the tinnitus and hearing loss returned. After reinstating the cariprazine to $3 \mathrm{mg}$ a day the tinnitus and hearing loss resolved again.

RESULTS: Psychiatric evaluation: Disheveled with postural tremor of both upper extremities. Pharyngeal dysarthria. Irritable and with expansive and labile affect. Severely impaired attention. Slow tangential thoughts, preoccupied with paranoia and suspiciousness. Paranoid delusions. Mental status examination: Memory testing: Immediate recall: 5 digits forwards and 3 digits backwards. Able to recall none of 4 objects in 3 minutes and 1 with reinforcement. Unable to spell the word "world." Similarities interpreted concretely. Calculation ability poor.

DISCUSSION: In the cochlea, as an inhibitory neurotransmitter, dopamine reduces sensitivity to auditory sensation (Langguth, 2009). Since ambient sounds are known to reduce tinnitus (masking), an antagonist at overstimulated dopamine receptors, cariprazine may act to reduce dopamine's effectiveness, reducing inhibition and thus enhancing perceived external sound. It may act as a 5-HT1A serotonin agonist, directly reducing tinnitus. With reduced tinnitus there is less of a distraction and thus enhanced hearing. Or its function may be through its neuroleptic effects; the tinnitus could be a manifestation of auditory hallucinations, through reduction of this noise cariprazine secondarily causes enhanced hearing. Further investigation into the use of cariprazine in those with intractable tinnitus is worthwhile.

FUNDING ACKNOWLEDGEMENTS: No funding.

\section{6}

\section{Improvement in Disease Severity in Patients With Treatment-Resistant Depression Following Treatment With Intranasal Esketamine}

Abigail Nash, MD, PhD'; May Shawi, PhD ${ }^{2}$; Jaskaran Singh, $\mathrm{MD}^{3}$; Ella Daly, $\mathrm{MD}^{4}$; Kimberly Copper, $M S^{4}$; Pilar Lim, PhD ${ }^{4}$; Rosanne Lane, MAS ; Jagadish Gogate, PhD ${ }^{4}$; Allitia DiBernardo, $\mathrm{MD}^{4}$; David Hough, $M D^{4}$; and Larry Alphs, $M D, P h D^{2}$

\footnotetext{
${ }^{1}$ Janssen Global Services, New Jersey

${ }^{2}$ Janssen Scientific Affairs, New Jersey

${ }^{3}$ Janssen R\&D, California

${ }^{4}$ Janssen R\&D, New Jersey
}

ABSTRACT: Background: Recognizing the importance not only of the clinician's opinion but also of the patient's experience and perspective, Sequenced Treatment Alternatives to Relieve Depression (STAR*D) utilized both clinician-reported and patient-reported outcomes in a large-scale multi-step study on antidepressant effectiveness in real-world settings. Both approaches indicate 